\title{
Application of Mild Therapeutic Hypothermia on Stroke: A Systematic Review and Meta-Analysis
}

\author{
Shaheen E. Lakhan and Fabricio Pamplona \\ Department of Biosciences, Global Neuroscience Initiative Foundation, Los Angeles, Panorama City, \\ P.O. Box 4832 CA 91412, USA \\ Correspondence should be addressed to Shaheen E. Lakhan, slakhan@gnif.org
}

Received 27 October 2011; Accepted 6 December 2011

Academic Editor: Chelsea S. Kidwell

Copyright ( $(2012$ S. E. Lakhan and F. Pamplona. This is an open access article distributed under the Creative Commons Attribution License, which permits unrestricted use, distribution, and reproduction in any medium, provided the original work is properly cited.

\begin{abstract}
Background. Stroke occurs due to an interruption in cerebral blood supply affecting neuronal function. Body temperature on hospital admission is an important predictor of clinical outcome. Therapeutic hypothermia is promising in clinical settings for stroke neuroprotection. Methods. MEDLINE/PubMed, CENTRAL, Stroke Center, and ClinicalTrials.gov were systematically searched for hypothermia intervention induced by external or endovascular cooling for acute stroke. NIH Stroke Scale (NIHSS) and modified Rankin Scale (mRS) were the main stroke scales used, and mortality was also reported. A meta-analysis was carried out on stroke severity and mortality. Results. Seven parallel-controlled clinical trials were included in the meta-analysis. Sample sizes ranged from 18 to 62 patients, yielding a total of 288 . Target temperature $\left(\sim 33^{\circ} \mathrm{C}\right)$ was reached within $3-4$ hours. Stroke severity (Cohen's $d=-0.17,95 \% \mathrm{CI}:-0.42$ to $0.08, P=0.32 ; I^{2}=73 \%$; $\left.\mathrm{Chi}^{2}=21.89, P=0.0001\right)$ and mortality $(\mathrm{RR}=1.60,95 \%$ CI: 0.93 to $2.78, P=0.11 ; I^{2}=0 \%$; $\mathrm{Chi}^{2}=2.88, P=0.72$ ) were not significantly affected by hypothermia. Discussion. Hypothermia does not significantly improve stroke severity; however, this finding should be taken with caution due to the high heterogeneity and limited number of included studies. No impact on mortality was observed.
\end{abstract}

\section{Background}

Stroke is a medical condition resulting from an interruption in the cerebral blood supply that affects neuronal function and is the leading cause of adult disability in the United States and Europe. Furthermore, a high mortality risk is observed in the stroke aftermath [1]. Some of the most common symptoms include disturbances of speech/language, motor, and sensory function. Direct causes of stroke include ischemia, thromboemoblism, and hemorrhage and its occurrence positively correlates with age, lipids, smoking pack years, and blood pressure [2].

As a single factor, body temperature on hospital admission is an important predictor of clinical outcome. The temperature is generally inversely correlated to post-acute stroke symptomatology. Fever in the early aftermath of symptoms onset predicts, worse clinical outcome [3-9] and very mild hypothermia $\left(<36.5^{\circ} \mathrm{C}\right)$ is associated with improved outcome and reduced mortality [7]. Therefore, at least in the investigational context, therapeutic hypothermia has emerged as a potentially promising neuroprotective therapy. The investigations of other neuroprotective therapies aimed to reduce the impact of stroke on the patient's quality of life have so far yielded limited results [10].

Further support to therapeutic hypothermia comes from studies employing animal models. Lowering body temperature has been shown to exert strong neuroprotection $[4,6]$, while elevation of body temperature extends brain damage $[4,11]$. Although the underlying mechanisms have never been completely understood, it is already well accepted that hypothermia decreases brain edema and mitigates the effects of brain ischemia/reperfusion, which are temperature dependent. In animal models, hypothermia also reduces infarct volume, decreases cerebral metabolism, reduces excitotoxicity due to synaptic glutamate overflow, and stabilizes the blood-brain barrier and neuronal membranes, while decreasing cerebral edema in animal models of acute stroke [12-15]. The efficacy of this 
therapeutic approach has a relatively restricted time-window [16].

In human patients, hypothermia is induced either by external cooling using traditional methods or by more recent endovascular cooling devices (reviewed in [17]). Surface cooling methods include air blankets, water mattresses, alcohol bathing, and ice packing. Patients in these conditions are usually intubated, ventilated, and heavily sedated to reduce shivering and bodily discomfort. The major advantage of external cooling is that it does not require advanced equipment to induce hypothermia but often requires many hours to reach target temperature and increases the chance of clinical complications such as shivering, pneumonia, and reactive brain edema (due to temperature overshooting). Hypothermia in awake patients has become feasible by the development of catheters covered with antithrombotic agents that can be inserted in the venous system allowing rapid heat exchange and faster cooling towards target body temperature [17]. Endovascular cooling allows skin warming during the procedure, therefore avoiding shivering and allowing a tighter temperature control. In the absence of endovascular cooling, shivering is prevented with medications like meperidine which also contributes to sedation [18]. Aside from shivering, another relevant side effect of hypothermia is the reduction in thrombolysis, which is prevented by anticlotting therapy such as supplementation of tissue plasminogen activator (tPA) $[19,20]$. Pneumonia is an indirect side-effect occurring as a consequence of intubation for long periods (required in the external cooling method). More modern devices for surface cooling (skin pads) may offer benefits over traditional techniques reducing the time to reach target temperature and increasing safety $[21,22]$.

In keeping with the variety of evidence showing that hypothermia may predict positive outcome on stroke patients, the induction of hypothermia in clinical settings is far from being a widely accepted and standardized procedure in stroke management. Therefore, the objective of this systematic review is to summarize all the relevant scientific literature coming from available clinical studies on the relation between low body temperature (hypothermia) and poststroke symptomatology. We aim to collect solid evidence to define whether induced hypothermia is a useful and safe procedure providing long-term benefits to acute stroke patients.

\section{Methods}

2.1. Identification, Inclusion, and Exclusion. A systematic search was performed in MEDLINE/PubMed (http://www .ncbi.nlm.nih.gov/pubmed/) and Cochrane Central Register of Controlled Trials (CENTRAL; http://onlinelibrary .wiley.com/) to find original articles with experimental data on the association of hypothermia and stroke, published before May 2011. The keywords "hypothermia" and "stroke" were used to search for words in the title or abstract of the articles. Additional references were included regarding the registered clinical trials presented in the Stroke Center (http://www.strokecenter.org/trials/) and NIH's ClinicalTrials.gov (http://clinicaltrials.gov/). The search was limited to studies written in the English language and performed in human adults. All titles, abstracts, and full papers of potentially relevant studies were assessed for eligibility based on predefined inclusion and exclusion criteria. Primary inclusion criteria: every original article with observational or experimental data written in English was considered potentially relevant for the meta-analysis. Self-controlled trials (quasi-experiments) were included as "trials" due to the similarity in the clinical outcomes assessed. Primary exclusion criteria: no data reported multiple treatments where we could not clearly access the impact of hypothermia, incompatible outcome measurement, case reports, review papers, and previous meta-analyses. Studies employing pharmacologically induced hypothermia, although important from the standpoint of the clinical practice, were intentionally excluded due to the potential confounding factors that could contribute to neuroprotection in stroke patients (e.g., anti-inflammatory effects). Nevertheless, two important randomized clinical trials of $[23,24]$ on the effects of paracetamol (acetaminophen) should be mentioned here. (See Additional file 1 for a Quality of Reporting of Metaanalyses (QUOROM) statement checklist in Supplementary Material available online at doi: 10.1155/2012/295906.)

2.2. Data Abstraction. Data were extracted independently by the authors and any disagreements were resolved by consensus.

2.3. Clinical Outcomes. From each selected study, data were extracted pertaining to study design, number of participants, population characteristics, intervention/group selection, clinical outcomes, and mortality rate. NIHSS and mRS were used as main stroke scales. The term "stroke" defined acute ischemic stroke or severe stroke. Only studies with fully accessible content and clear stroke diagnosis criteria were included in the final sample. Hypothermia was induced by external or internal cooling.

2.4. Statistical Analysis. In the meta-analysis, the effect size was calculated either as the standardized mean difference "Cohen's $d$ " for the stroke severity or as "risk ratio" for mortality. Both data sets were tested for heterogeneity $\left(I^{2}\right)$ and are expressed as mean (95\% CI). The statistics package Review Manager (RevMan) was used for the meta-analysis.

\section{Results}

The initial search using the keywords "hypothermia" and "stroke" in MEDLINE/PubMed yielded 1244 relevant references (see Figure 1). English language-written studies represented 1160 references. Previous review articles (206) and nonhuman studies (425) were excluded from the sample, leaving 502 abstracts to be read. Only 440 abstracts were in fact assessed, as 62 references had no abstract available (mostly comments and old articles). While reading the abstracts, we excluded (1) single-case studies, (2) references with data on newborns, children, elderly, or pregnant women, (3) studies with confounding factors, where the isolated effects of hypothermia on stroke could not be 


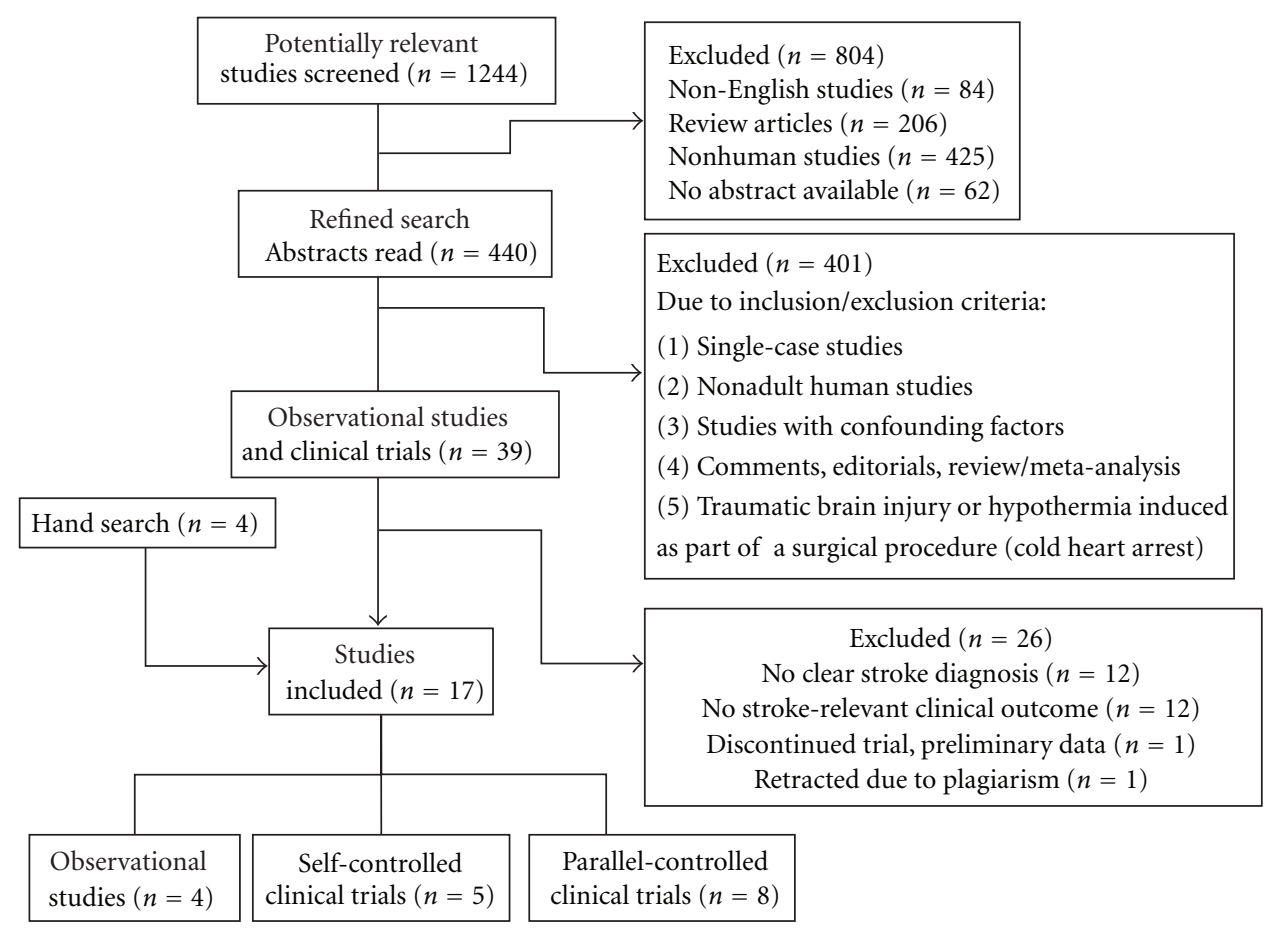

FIgURE 1: Flow chart of the systematic search.

assessed, (4) comments, editorials, or any review/metaanalysis, and (5) traumatic brain injury or hypothermia induced as part of a surgical procedure (cold heart arrest). In this process, 401 references were excluded, yielding a final count of 39 full articles to be carefully investigated. Twelve articles had no clear stroke criterion for diagnosis. Twelve additional references had no reference to strokerelevant clinical outcome. One registered clinical trial was discontinued and only preliminary data were accessible. One article was retracted due to plagiarism. Four nonredundant references were added by hand from clinical trial-specific databases. The data presented in Tables 1-3 represent a total of 17 articles, including 4 observational studies and 13 clinical trials ( 5 are self-controlled quasi-experiments and 8 are proper controlled clinical trials). The clinical study design guidelines from the Centre for Evidence-Based Medicine (CEBM, University of Oxford) were used to allocate the studies to the given categories.

3.1. Observational Studies. Two observational studies established the influence of body temperature on admission and the clinical outcome of stroke (Table 1). A prospective study grouping patients in "hypothermic" and "hyperthermia" according to the body temperature on admission (below and above $37^{\circ} \mathrm{C}$, consecutively) suggested that those patients whose body temperature was below the selected threshold showed reduced stroke severity and lower mortality both 3 and 60 months later [5]. An immediate criticism to this study is that it sets an acceptable threshold for hyperthermia, but it lacks distinction between hypothermia and normal body temperature. However, the evidence of association between the clinical outcome and admission temperature on stroke patients is confirmed by a better designed study with three temperature categories. This retrospective study created the category of "normothermia" $\left(36.5^{\circ} \mathrm{C}\right.$ to $\left.37.5^{\circ} \mathrm{C}\right)$ and defined hypothermia and hyperthermia relatively to this temperature range [9]. Stroke severity was not assessed in this study, but mortality both in-hospital and at 12 months after discharge was reduced by hypothermia. Another study established a clear inverse linear correlation between body temperature on admission and prospective clinical outcome of stroke [7]. For each $1^{\circ} \mathrm{C}$ increase in body temperature, the relative risk of poor outcome worsens more than two times. The overall picture is that body temperature in fact influences severity and mortality in stroke patients within a relatively narrow window $[8]$.

3.2. Self-Controlled Clinical Trials. Five studies represent the category of self-controlled clinical trials (Table 2). These studies include intervention (hypothermia was induced on the subjects) but the study design lacks a proper control group with another intervention or without any other medical procedure. In these studies, the clinical outcome is compared before and after the intervention, constituting a quasi-experiment with repeated measure (time series). The studies used either external cooling using fans and cold blankets or endovascular cooling to reduce the body temperature, but we will not make any distinction in the analysis. Regardless of the technique, therapeutic hypothermia was defined as the body temperature of $33-34^{\circ} \mathrm{C}$ (mild hypothermia) and applied within 3-6 hours of symptoms onset. Two studies assessed the effect of hypothermia on patients suffering from acute ischemic stroke $[25,26]$. In both cases, the individuals showed an improvement over 
TABLE 1: Observational studies (no intervention) of admission body temperature and mortality rate after acute stroke.

\begin{tabular}{|c|c|c|c|c|}
\hline \multicolumn{2}{|c|}{ Reference Study design } & \multirow{2}{*}{$\begin{array}{l}\text { Patients/groups } \\
390 \text { acute stroke patients }\end{array}$} & \multirow{2}{*}{$\begin{array}{l}\text { Stroke severity } \\
\text { Reduced body } \\
\text { temperature predicted } \\
\text { better clinical outcome }\end{array}$} & \multirow{2}{*}{$\begin{array}{l}\text { Mortality rate } \\
\text { Mortality was lower in patients with } \\
\text { mild hypothermia on admission }\end{array}$} \\
\hline [7] & $\begin{array}{l}\text { Prospective } \\
\text { Single-center }\end{array}$ & & & \\
\hline [9] & $\begin{array}{l}\text { Retrospective } \\
\text { Single-center }\end{array}$ & $\begin{array}{l}437 \text { patients; } 185 \text { hypothermic } \mathrm{BT} \\
\leq 36.5^{\circ} \mathrm{C} \text { versus } 199 \text { normothermic } \\
36.5^{\circ} \mathrm{C}>\mathrm{BT}<37.5^{\circ} \mathrm{C} \text { (and } 53 \\
\text { hyperthermic) }\end{array}$ & NR & $\begin{array}{l}0.1 \text { odds ratio in-hospital mortality } \\
(P=0.004) \\
0.8 \text { odds ratio at } 12 \text { months mortality } \\
(P=0.454, \mathrm{NS})\end{array}$ \\
\hline$[5]$ & $\begin{array}{l}\text { Prospective } \\
\text { Single-center }\end{array}$ & $\begin{array}{l}390 \text { acute stroke patients; } 179 \\
\text { hypothermic } \mathrm{BT} \leq 37^{\circ} \mathrm{C} \text { versus } 211 \\
\text { hyperthermic } \mathrm{BT}>37^{\circ} \mathrm{C}\end{array}$ & $\begin{array}{l}-21 \%(P<0.001) \\
\text { Assessed by SSS }\end{array}$ & $\begin{array}{l}-28 \% \text { at } 3 \text { months }(P<0.001) \\
-54 \% \text { at } 60 \text { months }(P<0.006)\end{array}$ \\
\hline [8] & $\begin{array}{l}\text { Prospective } \\
\text { Single-center }\end{array}$ & 100 acute ischemic stroke patients & NR & $\begin{array}{l}\text { Mortality was higher in hyperthermic } \\
\left(>37.5^{\circ} \mathrm{C}\right) \text { patients and lower in } \\
\text { hypothermic patients }\left(<36.5^{\circ} \mathrm{C}\right) \text { than } \\
\text { those with regular temperature } \\
0 / 8 \text { hypothermic, } 17 / 30 \text { hyperthermic } \\
\text { versus } 6 / 62 \text { normothemic patients died }\end{array}$ \\
\hline
\end{tabular}

BT: body temperature; NR: not reported; NS: not statistically significant; SSS: Scandinavian Stroke Scale.

time, leaving the hospital with lower NIHSS compared to the admission. Just one of these studies reported mortality (roughly 10\%). Three other studies assessed the effects of therapeutic hypothermia on severe ischemic stroke [2729]. These studies induced hypothermia using noninvasive methods and reached target temperature range. The clinical state of the severe ischemic stroke patients improved after hypothermia as far as intracranial pressure, cerebral blood flow, and metabolic rate of oxygen are concerned. The mortality rate was higher than that for patients with acute stroke (between 33\% and 44\%). Although suggesting effectiveness of therapeutic hypothermia, due to the lack of a proper control groups, the results of these quasi-experiments are not enough to assume that hypothermia would in fact influence stroke.

3.3. Parallel-Controlled Clinical Trials. Eight studies employed proper controlled clinical trials design to investigate the effects of mild therapeutic hypothermia on stroke. Half of them were nonrandomized and two were double-blinded. Hypothermia was induced by external [20,30, 31] or endovascular $[19,32,33]$ devices, or both $[34,35]$. The target temperature was defined as $33^{\circ} \mathrm{C}$ for hypothermia and $\sim 36.5^{\circ} \mathrm{C}$ for normothermia. Three of them investigated acute ischemic stroke patients, showing just mild and transient improvement on stroke severity. No consistent differences were observed on stroke symptoms (using standardized clinical scales) at discharge, after 7-30 days or 3 months after the intervention. Only one study showed improvement in NIHSS [35]. This very study reported differences in mortality rate for the combination of hypothermia and craniectomy compared to craniectomy alone; however mortality rates were still lower than historical stroke controls without any therapeutic attempt [35]. The other studies either did not show difference on mortality rate or did not report this parameter.
Randomized controlled clinical trials are the gold standard design of clinical studies, and all relevant suggestion of potential clinical application of a given technique/procedure should be validated by a large randomized clinical trial before being largely implemented. For the topic of "hypothermia and stroke", to the best of our knowledge, four randomized trials have been carried out so far [19, 30, 33, 34], just two of them using a double-blind design [30, 33]. Open label studies showed divergent findings [19, 20, 31, 32, 34, 35]. One study comparing the effects of hypothermia versus craniectomy reported a positive impact of hypothermia on stroke severity with a four-point difference in NIHSS (21 versus 17; $P<0.0002$ ) [35]. The intervention was performed within $24 \mathrm{~h}$ of symptoms onset, and apparently all patients reached the target temperature of $33^{\circ} \mathrm{C}$. In the same light, the combination of craniectomy plus hypothermia applied immediately after surgery showed a trend towards positive impact on stroke severity against craniectomy alone [34]. This trend was observable in the NIHSS $(P=0.08)$, but not on Barthel index (BI) or mRS. In general, more "pure" comparisons of hypothermia versus no intervention failed to show clinical difference using mean lesion growth, intracranial pressure, NIHSS, or mRS as parameters [19, 20, $31,32]$. The only variable that seems to be affected in the hypothermia group compared to control is the volume of brain edema measured at the aftermath of the intervention (up to 2 days later); however, the difference vanished at 30day follow-up [32]. There was no apparent improvement in the clinical outcome with earlier versus late intervention considering time of symptoms onset. The open label study employing the earliest intervention latency (within 5 to $8 \mathrm{~h}$ of stroke symptoms onset) failed to find therapeutic benefit of hypothermia in the total duration of hospitalization and stroke severity (mRS) at the 3-month follow-up [20].

Two studies employed a superior design for the clinical trial; using randomized blinded designs [30, 33], they provide an interesting comparison because both are of 
TABLE 2: Self-controlled clinical trials (quasi experiment) of feasibility and efficacy of hypothermic intervention for stroke.

\begin{tabular}{|c|c|c|c|c|c|c|}
\hline Reference & Study design & Intervention & Patients & $\begin{array}{l}\text { Hypothermia } \\
\text { induction }\end{array}$ & $\begin{array}{l}\text { Impact on stroke } \\
\text { severity }\end{array}$ & Mortality \\
\hline$[29]^{\mathrm{a}}$ & $\begin{array}{l}\text { Nonrandomized, } \\
\text { open label, } \\
\text { self-controlled, } \\
\text { single-center } \\
\text { (quasi-experiment) }\end{array}$ & $\begin{array}{l}\text { Mild hypothermia } \\
\text { induced by external } \\
\text { cooling within } 4 \text { to } \\
24 \text { hours of } \\
\text { symptoms onset. } \\
\text { Target temperature } \\
\left(33^{\circ} \mathrm{C}\right)\end{array}$ & $\begin{array}{l}25 \text { patients with } \\
\text { severe ischemic } \\
\text { stroke, median SSS } \\
24, \text { mean GCS } 9\end{array}$ & $\begin{array}{l}\text { Target temperature } \\
\text { reached in } 3.5 \text { to } \\
6.2 \mathrm{~h} \text {. } \\
\text { Does not clear } \\
\text { report the \% of } \\
\text { patients reaching } \\
\text { target temperature, } \\
\text { apparently all of } \\
\text { them. }\end{array}$ & $\begin{array}{l}\text { Mean initial ICP } \\
20.9 \pm 12.4 \mathrm{mmHg} \\
\text { and reduced to } \\
13.4 \pm 8.3 \mathrm{mmHg} \\
\text { during hypothermia } \\
(P<0.05) \\
\text { Surviving patients } \\
\text { displayed SSS } 29 \\
\text { after } 4 \text { weeks and } 38 \\
\text { three months after } \\
\text { stroke }\end{array}$ & $\begin{array}{l}11 / 25 \\
\text { patients died }\end{array}$ \\
\hline$[28]^{\mathrm{b}}$ & $\begin{array}{l}\text { Nonrandomized, } \\
\text { open label, } \\
\text { self-controlled, } \\
\text { single-center } \\
\text { (quasi-experiment) }\end{array}$ & $\begin{array}{l}\text { Mild hypothermia } \\
\text { induced by external } \\
\text { cooling within } 6 \\
\text { hours of symptoms } \\
\text { onset. } \\
\text { Target temperature } \\
\left(33^{\circ} \mathrm{C}\right)\end{array}$ & $\begin{array}{l}20 \text { patients with } \\
\text { severe ischemic } \\
\text { stroke, median SSS } \\
27, \text { mean GCS } 9\end{array}$ & $\begin{array}{l}\text { All patients reached } \\
\text { target temperature } \\
\text { in about } 5-6 \mathrm{~h} .\end{array}$ & $\begin{array}{l}\text { Mean SSS was } \\
31.3 \pm 8.3 \text {, mean } \\
\text { mRS } 3 \text { and mean BI } \\
65 \text { four weeks after } \\
\text { stroke } \\
\text { ICP decreased with } \\
\text { initiation of } \\
\text { hypothermia }\end{array}$ & $\begin{array}{l}8 / 20 \text { patients } \\
\text { died }\end{array}$ \\
\hline$[27]$ & $\begin{array}{l}\text { Nonrandomized, } \\
\text { open label, } \\
\text { self-controlled, } \\
\text { single-center } \\
\text { (quasi-experiment) }\end{array}$ & $\begin{array}{l}\text { Mild hypothermia } \\
\text { induced by external } \\
\text { cooling as soon as } \\
\text { possible (no } \\
\text { mention of time } \\
\text { delay after } \\
\text { symptoms onset). } \\
\text { Target temperature } \\
\left(33-34^{\circ} \mathrm{C}\right)\end{array}$ & $\begin{array}{l}6 \text { patients with } \\
\text { severe ischemic } \\
\text { stroke }\end{array}$ & $\begin{array}{l}\text { Mean duration of } \\
\text { hypothermia } 63.5 \mathrm{~h} \\
\text { Does not clear } \\
\text { report the \% of } \\
\text { patients reaching } \\
\text { target temperature, } \\
\text { apparently all of } \\
\text { them. }\end{array}$ & $\begin{array}{l}\text { Decreased } \mathrm{CMRO} 2 \text {, } \\
\text { transiently reduced } \\
\mathrm{CBF} \text { and controlled } \\
\mathrm{ICP}\end{array}$ & $\begin{array}{l}2 / 6 \text { patients } \\
\text { died of } \\
\text { intractable } \\
\text { intracranial } \\
\text { hypertension }\end{array}$ \\
\hline
\end{tabular}

\section{Temperature}

dropped from

$37.1 \pm 0.7^{\circ} \mathrm{C}$ by a

maximum of

$1.6 \pm 0.3^{\circ} \mathrm{C}$

$(P<0.005)$ at

Nonrandomized, open label, self-controlled, single-center (quasi-experiment) induced by

endovascular device 10 patients with within $3 \mathrm{~h}$ of symptoms onset Target temperature $\left(33-34^{\circ} \mathrm{C}\right)$
Mild hypothermia

\section{acute ischemic} stroke NIHSS 4-12
$52 \pm 16$ min after

hypothermia

induction.

Does not clear

report the $\%$ of

patients reaching

target temperature, apparently all of them.

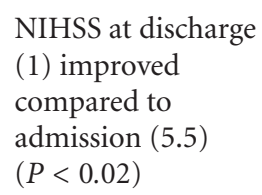

were not cooled due discharge
2/18 patients died
Mild hypothermia induced by ext $n=10)$ or $(n=8)$ cooling methods within $6 \mathrm{~h}$ of symptoms onset.
Target temperature $\left(33-34.5^{\circ} \mathrm{C}\right)$
18 acute stroke
patients NIHSS $\geq 8$ 
TABLE 3: Parallel-controlled clinical trials of feasibility and efficacy of hypothermic intervention for stroke.

\begin{tabular}{|c|c|c|c|c|c|c|}
\hline Reference & Study design & Intervention & Patients & $\begin{array}{l}\text { Hypothermia } \\
\text { induction }\end{array}$ & $\begin{array}{l}\text { Impact on stroke } \\
\text { severity }\end{array}$ & Mortality \\
\hline$[30]$ & $\begin{array}{l}\text { Randomized, } \\
\text { double-blinded, } \\
\text { parallel control, } \\
\text { multicenter }\end{array}$ & $\begin{array}{l}\text { Mild hypothermia } \\
\text { induced by } \\
\text { external cooling } \\
\text { during craniotomy } \\
\text { surgery } \\
\text { Target temperature } \\
\left(33.5^{\circ} \mathrm{C}\right)\end{array}$ & $\begin{array}{l}62 \text { patients with } \\
\text { intracranial } \\
\text { aneurysm ( } 33 \\
\text { hypothermia } \\
\text { versus } 29 \\
\text { normothermic } \\
\text { controls) } \\
\text { (hemorrhagic } \\
\text { excluded) }\end{array}$ & $\begin{array}{l}29 / 33 \text { patients were } \\
\text { effectively cooled } \\
(88 \%) \text { within } 1.0^{\circ} \mathrm{C} \text { of } \\
\text { target temperature. } \\
\text { Hypothermics } \\
\text { reached } 33.7^{\circ} \mathrm{C} \text { versus } \\
\text { normothermic } \\
\text { controls } 36.6^{\circ} \mathrm{C} \\
(P<0.0001)\end{array}$ & $\begin{array}{l}\text { No difference in NIHSS } \\
\text { at } 24 \text { and } 72 \mathrm{~h} \text { after } \\
\text { surgery. }\end{array}$ & $\begin{array}{l}2 / 33 \text { patients died } \\
\text { in the } \\
\text { hypothermic } \\
\text { group versus } 0 / 29 \\
\text { in the control } \\
\text { group }\end{array}$ \\
\hline [20] & $\begin{array}{l}\text { Nonrandomized, } \\
\text { open label, } \\
\text { parallel control, } \\
\text { single-center }\end{array}$ & $\begin{array}{l}\text { Mild hypothermia } \\
\text { induced by } \\
\text { external cooling } \\
\text { within } 5-8 \mathrm{~h} \text { of } \\
\text { symptoms onset. } \\
\text { Target temperature } \\
\left(32 \pm 1^{\circ} \mathrm{C}\right)\end{array}$ & $\begin{array}{l}19 \text { acute stroke } \\
\text { patients NIHSS } \\
>8(10 \\
\text { hypothermia } \\
\text { versus } 9 \\
\text { normothermic } \\
\text { controls })\end{array}$ & $\begin{array}{l}\text { Target temperature } \\
\left(32 \pm 1^{\circ} \mathrm{C}\right) \text { reached in } \\
3.5 \pm 1.5 \mathrm{~h} \text { and } \\
\text { maintained for } \\
22.8 \pm 8 \mathrm{~h} \text {. } \\
\text { All patients in the } \\
\text { hypothermia group } \\
\text { reached target } \\
\text { temperature }\end{array}$ & $\begin{array}{l}\text { mRS at } 3 \text { months: } \\
3.1 \pm 2.3 \\
\text { (hypothermia) versus } \\
4.2 \pm 1.6 \text { (control) (NS) } \\
\text { Hospital stay: } 10.9 \pm \\
6.7 \text { (hypothermia) } \\
\text { versus } 10.4 \pm 5.9 \\
\text { (control) (NS) }\end{array}$ & $\begin{array}{l}3 / 10 \text { hypothermic } \\
\text { patients died } \\
\text { versus } 2 / 9 \text { deaths } \\
\text { in the control } \\
\text { group }\end{array}$ \\
\hline [35] & $\begin{array}{l}\text { Nonrandomized, } \\
\text { open label, } \\
\text { parallel control, } \\
\text { single-center }\end{array}$ & $\begin{array}{l}\text { Mild hypothermia } \\
\text { induced by } \\
\text { external cooling } \\
(n=11) \text { or } \\
\text { endovascular } \\
\text { cooling }(n=8) \\
\text { within } 24 \mathrm{~h} \text { of } \\
\text { symptoms onset } \\
\text { Target temperature } \\
\left(33^{\circ} \mathrm{C}\right)\end{array}$ & $\begin{array}{l}36 \text { acute ischemic } \\
\text { stroke patients } \\
\text { NIHSS }>15 \text { ( } 19 \\
\text { hypothermia } \\
\text { versus } 17 \\
\text { hemicraniectomy) }\end{array}$ & $\begin{array}{l}\text { Target temperature } \\
\left(33^{\circ} \mathrm{C}\right) \text { reached in } \\
4 \pm 1 \mathrm{~h} \text {. } \\
\text { Does not clear report } \\
\text { the } \% \text { of patients } \\
\text { reaching target } \\
\text { temperature, } \\
\text { apparently all of them }\end{array}$ & $\begin{array}{l}\text { NIHSS } 17 \\
\text { (hypothermia) versus } \\
21 \text { (hemicraniectomy) } \\
(P<0.002)\end{array}$ & $\begin{array}{l}9 / 19 \text { hypothermic } \\
\text { patients died } \\
\text { versus } 2 / 17 \\
\text { hemicraniectomy } \\
(P<0.02)\end{array}$ \\
\hline [19] & $\begin{array}{l}\text { Randomized, } \\
\text { open label, } \\
\text { parallel control, } \\
\text { multicenter }\end{array}$ & $\begin{array}{l}\text { Mild hypothermia } \\
\text { induced by } \\
\text { endovascular } \\
\text { device less than } 9 \\
\text { hours of stroke } \\
\text { symptoms onset. } \\
\text { Target temperature } \\
\left(33^{\circ} \mathrm{C}\right) \\
\end{array}$ & $\begin{array}{l}40 \text { patients NIHSS } \\
>8(18 \\
\text { hypothermia } \\
\text { versus } 22 \\
\text { normothermic } \\
\text { controls) }\end{array}$ & $\begin{array}{l}13 / 18 \text { patients were } \\
\text { effectively cooled } \\
(72 \%), \text { reaching } \\
\text { target temperature in } \\
77 \pm 44 \text { min. Mean } \\
\text { time from stroke } \\
\text { onset to cooling was } \\
8 \mathrm{~h} 59 \pm 2 \mathrm{~h} 52\end{array}$ & $\begin{array}{l}\text { NIHSS, mRS, and } \\
\text { mean lesion growth } \\
\text { were similar between } \\
\text { groups (NS) }\end{array}$ & $\begin{array}{l}5 / 18 \text { patients died } \\
\text { in the } \\
\text { hypothermia } \\
\text { group; } 4 / 22 \\
\text { patients died in } \\
\text { the control group }\end{array}$ \\
\hline$[34]$ & $\begin{array}{l}\text { Randomized, } \\
\text { open label, } \\
\text { parallel control, } \\
\text { single-center }\end{array}$ & $\begin{array}{l}\text { Mild hypothermia } \\
\text { induced by internal } \\
(n=10) \text { or } \\
\text { external cooling } \\
(n=2) \\
\text { immediately after } \\
\text { surgery. } \\
\text { Target temperature } \\
\left(35^{\circ} \mathrm{C}\right)\end{array}$ & $\begin{array}{l}25 \text { severe ischemic } \\
\text { stroke patients }(12 \\
\text { craniectomy }+ \\
\text { hypothermia } \\
\text { versus } 13 \\
\text { craniectomy). } \\
\text { Patients in the } \\
\text { craniectomy } \\
\text { control group } \\
\text { were kept } \\
\text { normothermic } \\
\left(>37.5^{\circ} \mathrm{C}\right)\end{array}$ & $\begin{array}{l}\text { Target temperature } \\
\text { reached within } \\
2 \pm 1 \mathrm{~h} \text {. } \\
\text { Does not clear report } \\
\text { the } \% \text { of patients } \\
\text { reaching target } \\
\text { temperature, } \\
\text { apparently all of them }\end{array}$ & $\begin{array}{l}\text { Trend towards clinical } \\
\text { improvement for the } \\
\text { combined treatment } \\
\text { NIHSS }(10 \pm 1 \text { versus } \\
11 \pm 3, P=0.08) \text { and } \\
\text { BI }(81 \pm 14 \text { versus } \\
70 \pm 17, P<0.1) \text {. } \\
\text { No significant } \\
\text { difference were } \\
\text { observed in mRS }(2 \pm 1 \\
\text { versus } 3 \pm 1, P=0.18) \\
\text { after } 6 \text { months }\end{array}$ & $\begin{array}{l}\text { 1/12 hypothermic } \\
\text { patients died } \\
\text { versus } 2 / 13 \\
\text { patients in the } \\
\text { craniectomy } \\
\text { group }\end{array}$ \\
\hline$[32]$ & $\begin{array}{l}\text { Nonrandomized, } \\
\text { open label, } \\
\text { parallel control, } \\
\text { multicenter }\end{array}$ & $\begin{array}{l}\text { Mild hypothermia } \\
\text { induced by } \\
\text { endovascular } \\
\text { cooling within } 12 \mathrm{~h} \\
\text { of symptoms onset } \\
\text { Target temperature } \\
\left(33^{\circ} \mathrm{C}\right)\end{array}$ & $\begin{array}{l}18 \text { acute ischemic } \\
\text { stroke patients ( } 7 \\
\text { effectively cooled } \\
\text { versus } 11 \\
\text { normothermic } \\
\text { controls) }\end{array}$ & $\begin{array}{l}7 / 18 \text { patients were } \\
\text { effectively cooled } \\
(39 \%) \text { and tolerated } \\
\text { up to } 33.5 \pm 0.6^{\circ} \mathrm{C} \\
\text { versus normothermia } \\
\text { in the control group } \\
35.7 \pm 0.7^{\circ} \mathrm{C} \\
(P<0.001) \text {. } \\
\text { Target temperature } \\
\left(33^{\circ} \mathrm{C}\right)\end{array}$ & $\begin{array}{l}\text { Difference in brain } \\
\text { edema during } 36-48 \mathrm{~h} \\
(P<0.01) \text { that } \\
\text { vanished after } 30 \text { days. } \\
\text { No significant } \\
\text { difference in NIHSS at } \\
\text { catheter removal, and } \\
\text { after } 7 \text { days. No } \\
\text { difference in NIHSS or } \\
\text { mRS after } 30 \text { days }\end{array}$ & NR \\
\hline
\end{tabular}


TABle 3: Continued.

\begin{tabular}{|c|c|c|c|c|c|c|}
\hline Reference & Study design & Intervention & Patients & $\begin{array}{l}\text { Hypothermia } \\
\text { induction }\end{array}$ & $\begin{array}{l}\text { Impact on stroke } \\
\text { severity }\end{array}$ & Mortality \\
\hline$[31]$ & $\begin{array}{l}\text { Nonrandomized, } \\
\text { open label, parallel } \\
\text { control, } \\
\text { single-center }\end{array}$ & $\begin{array}{l}\text { Mild hypothermia } \\
\text { induced by } \\
\text { external cooling } \\
\text { within } 10 \text { to } 24 \mathrm{~h} \\
\text { of symptoms onset } \\
\text { Target temperature } \\
\left(33^{\circ} \mathrm{C}\right)\end{array}$ & $\begin{array}{l}30 \text { severe stroke } \\
\text { patients, mean SSS } \\
17-17.5(10 \\
\text { hypothermics } \\
\text { versus } 20 \\
\text { normothermic } \\
\text { controls) }\end{array}$ & $\begin{array}{l}\text { Does not clear } \\
\text { report the \% of } \\
\text { patients reaching } \\
\text { target temperature, } \\
\text { apparently all of } \\
\text { them }\end{array}$ & $\begin{array}{l}\text { Intracranial pressure } \\
\text { did not differ between } \\
\text { groups }(P=0.41) \\
\text { MMP9 (biomarker of } \\
\text { blood brain barrier } \\
\text { breakdown) } \\
\text { marginally lower in } \\
\text { hypothermics } \\
(P=0.05)\end{array}$ & NR \\
\hline [33] & $\begin{array}{l}\text { Randomized, } \\
\text { double-blinded, } \\
\text { parallel control, } \\
\text { multicenter }\end{array}$ & $\begin{array}{l}\text { Mild hypothermia } \\
\text { induced by } \\
\text { endovascular } \\
\text { device within } 0-3 \\
\text { or 3-6 hours of } \\
\text { symptoms onset. } \\
\text { Target temperature } \\
\left(33^{\circ} \mathrm{C}\right)\end{array}$ & $\begin{array}{l}58 \text { patients with } \\
\text { acute stroke } \\
\text { symptoms (NIHSS } \\
7 \geq \text { ) } \\
28 \text { hypothermia } \\
\text { versus } 30 \\
\text { normothermic } \\
\text { controls. } \\
\text { Also compared the } \\
\text { latency time from } \\
\text { symptoms onset } \\
\text { and the } \\
\text { combination with } \\
\text { thrombolytic } \\
\text { therapy }\end{array}$ & $\begin{array}{l}\text { Target temperature } \\
\text { was reached in } \\
20 / 28 \text { patients } \\
(71.4 \%) \text { in about } \\
67 \text { min (median } \\
\text { time). } \\
\text { Patients not } \\
\text { reaching target } \\
\text { temperature had a } \\
\text { mean temperature } \\
\text { of } 33.4 \pm 0.6^{\circ} \mathrm{C} \text {. }\end{array}$ & $\begin{array}{l}\text { Difference in NIHSS } \\
\text { at } 24 \text { h due to } \\
\text { sedation with } \\
\text { meperidine: } 17.0 \pm 8.9 \\
\text { in the hypothermic } \\
\text { group versus } \\
11.1 \pm 8.1 \text { in the } \\
\text { controls }(P<0.02) \text {. } \\
\text { The NIHSS was } \\
\text { equivalent in both } \\
\text { groups at } 30 \text { days } \\
\text { ( } 8.0 \pm 6.5 \text { versus } \\
5.0 \pm 4.1) \text { and } 90 \text { days } \\
\text { ( } 6.3 \pm 6.6 \text { versus } \\
3.8 \pm 3.0) \text {. No } \\
\text { difference in mRS at } \\
90 \text { days. } \\
\text { Thrombolytic therapy } \\
\text { (tPA) did not } \\
\text { influence clinical } \\
\text { outcome or } \\
\text { occurrence of adverse } \\
\text { events }\end{array}$ & $\begin{array}{l}6 / 28 \text { patients died in } \\
\text { the hypothermic } \\
\text { group versus } 5 / 30 \text { in } \\
\text { the control group } \\
\text { Pneumonia occurred } \\
\text { more frequently in } \\
\text { hypothermic patients } \\
(7 / 28) \text { than controls } \\
(2 / 30)(P<0.05)\end{array}$ \\
\hline
\end{tabular}

ICP: intracranial pressure; MMP9: matrix metalloproteinase 9; mRS: modified Rankin scale; NIHSS: NIH Stroke Scale NR: not reported; NS: not statistically significant; SSS: Scandinavian Stroke Scale.

reasonable size and employ different therapeutic approaches (external versus endovascular cooling). One of them compared the effect of hypothermia versus normothermia in 62 patients undergone craniectomy, from which $88 \%$ were effectively cooled [30]. No differences in stroke symptoms (NIHSS) at $24 \mathrm{~h}$ or $72 \mathrm{~h}$ and mortality were observed [30]. The more recent study confirms this earlier observation employing an endovascular cooling method [33]. A smaller proportion of patients reached target temperature in this study $(71.4 \%)$, but all of them stayed within $0.5-1^{\circ} \mathrm{C}$ of the target. NIHSS was worse at $24 \mathrm{~h}$ with endovascular cooling (17.0 versus $11.1 ; P<0.02)$ likely secondary to the sedative effects of meperidine in attempt to reduce shivering. Nevertheless, no difference was observed at day 30 or 90 after intervention per NIHSS or mRS [33]. This study also compared the time window of the hypothermic intervention $(<3 \mathrm{~h}$ versus $3-6 \mathrm{~h}$ of symptoms onset) and evaluated the impact of adjuvant thrombolytic therapy. There was no apparent difference regarding time of intervention and thrombolytic therapy (tPA) on clinical outcome or rate of adverse events [33].
Mortality was comparable between hypothermia and controls for all the studies. The two randomized blinded clinical trial of mild therapeutic hypothermia on stroke enrolled 120 stroke patients in total and found no difference between groups $[30,33]$. As for the adverse events, pneumonia occurred more frequently in hypothermic patients than in controls $(P<0.05)$, as expected [33]. Fortunately, the occurrence of pneumonia did not impact the improvement on stroke symptoms as measured by the mRS.

3.4. Meta-Analysis. The results of stroke severity and mortality were reported as meta-analysis using a Forest plot. Clinical outcomes of the hypothermia intervention versus control were pooled together and represented in Figure 2; mortality rates of the same studies are represented in Figure 3. The study of Horstmann et al., 2008, was not included in the analysis because it did not assess the same outcomes (i.e., therapeutic outcome assessed by standardized stroke scale and mortality rate). As analyzed by the global data, hypothermia did not exert a great influence on stroke clinical outcome, with a pooled effect size of $d=-0.17$ 


\begin{tabular}{|c|c|c|c|c|c|c|c|c|c|c|c|c|c|}
\hline \multirow[b]{2}{*}{ Study or subgroup } & \multicolumn{3}{|c|}{ Hypothermia } & \multicolumn{3}{|c|}{ Control } & \multicolumn{3}{|c|}{ Std. mean difference } & \multirow{2}{*}{\multicolumn{3}{|c|}{$\begin{array}{l}\text { Std. mean difference } \\
\text { IV, fixed, } 95 \% \text { CI }\end{array}$}} & \\
\hline & Mean & $\mathrm{SD}$ & Total & Mean & $\mathrm{SD}$ & Total & Weight & IV, fixed, 95\% CI & Year & & & & \\
\hline Hindman 1999 & 1 & 1.5 & 33 & 1 & 1 & 29 & $25.4 \%$ & $0.00[-0.5,0.50]$ & 1999 & & & & \\
\hline Krieger 2001 & 3.1 & 2.3 & 10 & 4.2 & 1.6 & 9 & $7.5 \%$ & $-0.53[-1.44,0.39]$ & 2001 & & & & \\
\hline Georgiadis 2002 & 17 & 2 & 19 & 21 & 3 & 17 & $11 \%$ & $-1.55[-2.31,-0.80]$ & 2002 & & & & \\
\hline De Georgia 2004 & 19.2 & 1.9 & 18 & 20.3 & 4.5 & 22 & $16.1 \%$ & $-0.30[-0.93,0.33]$ & 2004 & & & & \\
\hline Els 2006 & 10 & 1 & 12 & 11 & 3 & 13 & $10 \%$ & $-0.43[-1.22,0.37]$ & 2006 & & & & \\
\hline Guluma 2008 & 13.3 & 11 & 11 & 12.3 & 8.5 & 7 & $7 \%$ & $0.09[-0.85,1.04]$ & 2008 & & & & \\
\hline Hemmen et al. 2010 & 8 & 6.5 & 28 & 5 & 4.1 & 30 & $22.9 \%$ & $0.55[0.02,1.07]$ & 2010 & & & - & \\
\hline Total $(95 \% \mathrm{CI})$ & & & 131 & & & 127 & $100 \%$ & $-0.17[-0.42,0.08]$ & & & & & \\
\hline \multicolumn{10}{|c|}{$\begin{array}{l}\text { Heterogeneity: } \mathrm{Chi}^{2}=21.89, d f=6(P=0.001) ; I^{2}=73 \% \\
\text { Test for overall effect: } Z=1.32(P=0.19)\end{array}$} & -2 & -1 & 1 & 2 \\
\hline
\end{tabular}

FIgURE 2: Forest plot illustrating the meta-analysis of the clinical outcome (stroke severity) presented in the controlled clinical trials of hypothermia and stroke.

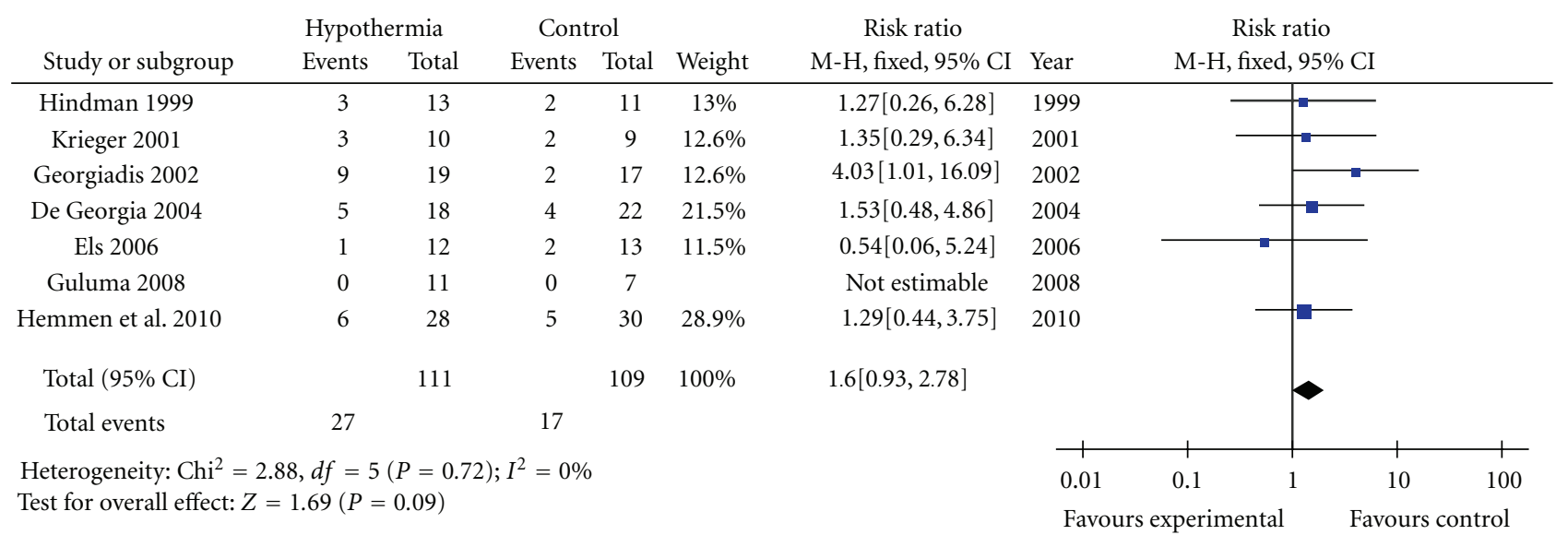

FIGURE 3: Forest plot illustrating the meta-analysis of the mortality rate presented in the controlled clinical trials of hypothermia and stroke.

(95\% CI: -0.42 to $0.08 ; P=0.32$ ). The mortality was similar for stroke patients who underwent hypothermia and controls, with a pooled risk ratio of 1.60 (95\% CI: 0.93 to 2.78; $P=0.11)$. The results of the effect size meta-analysis for the clinical outcome should be taken with caution due to high inconsistency/heterogeneity $\left(I^{2}=73 \%\right.$; $\mathrm{Chi}^{2}=21.89$, $P=0.0001)$; the results of the relative risk meta analysis for the patients mortality are perfectly consistent $\left(I^{2}=0 \%\right.$; $\mathrm{Chi}^{2}=2.88, P=0.72$ ).

\section{Discussion}

Mild alterations in body temperature are strong predictors of clinical outcome in stroke. Previous studies have established an inverse linear correlation between temperature on hospital admission and stroke severity, infarct size, and mortality [7]. Patients presenting fever on hospital admission have worse symptomatology than those presenting regular temperature [3]. The observational studies fully assessed during the elaboration of this systematic review also suggested this association between stroke severity/mortality rate and body temperature. One study showed a significant improvement $(\sim 21 \%)$ in stroke severity in hypothermic patients compared to hyperthermic ones, with long-term consequences observed in the 60-month follow-up phase [5]. Nevertheless, it must be conceived that the temperature threshold established for hypothermia was quite high $\left(\leq 37^{\circ} \mathrm{C}\right)$. Therefore, in fact, they observed worsening effects of hyperthermia rather than beneficial effects of hypothermia. The second study overcame this issue by classifying the patients in three categories. Hypothermia in this case was defined as $\leq 36.5^{\circ} \mathrm{C}$, hyperthermia as $\geq 37.5^{\circ} \mathrm{C}$, and the temperatures in-between were considered "normothermia". In this case, there was a transient improvement in the in-hospital mortality, which was not observed in the one-year follow-up phase [36]. Taken together, the observational studies suggested that the clinical outcome of stroke patients is largely influenced by the temperature on admission. The studies are limited in number (2) and do not provide conclusive evidence on the matter, but their results encourage careful analysis of the clinical trials.

Some interesting studies addressed the feasibility of procedures to induce therapeutic hypothermia on stroke patients. While they did not fulfill the criteria to be included 
in the tables of the present systematic review, it is worth mentioning them to describe the diversity and evolution of therapeutic hypothermia methods. In general, the techniques employ either conventional external cooling (using fans, cold blankets, and ice) or endovascular probes [37]. Feasibility studies are important because despite the clear relation between body temperature and stroke outcome, viable application of therapeutic hypothermia in clinical settings is often limited by comfort issues. One of the most frequent challenges is the autonomic shivering response, which tends to complicate the cooling process [38]. This bodily shivering response can be prevented by pharmacological means (antishivering drugs) and is mostly evoked by the external cooling procedure, reason by which the standard method of external cooling is being slowly substituted by methods of endovascular cooling that presumably allow faster brain cooling [17]. Anecdotal evidence suggests that intravascular cooling method might be better tolerated than surface cooling in awake patients, although the two have never been directly compared in a randomized trial.

The standard procedure of external cooling requires sedating and intubating patients and provides surface cooling with cooling blanket and/or alcohol/ice bath [39]. Although relatively safe, one relevant potential complication of external cooling is ventilator associated pneumonia, and the risk of occurrence is proportional to hypothermia duration and patient age [39]. One attempt to increase safety in the external cooling procedure was the use of slow controlled rewarming at the end of hypothermia [40]. Nevertheless, endovascular cooling using catheters introduced in the inferior vena cava is the current technique of choice, since it can be done in awake patients, rapidly, and precisely decreasing the core body temperature [41]. Endovascular cooling does not need intubation/sedation or neuromuscular blockade and often induces minimal discomfort or shivering. Surface warming with heating blanket can also be used in parallel to endogenous cooling, plus buspirone/meperidine as prophylactic pharmacological antishivering agents [38]. Another interesting initiative was the development of a cooling helmet, which induces fast and selective brain cooling, providing regional hypothermia with minimal systemic complications [36]. Nevertheless, the use of cooling helmet has not been consistently replicated and it is unclear how deep brain structures might be affected as the temperature was measured just $0.8 \mathrm{~cm}$ under the cortical surface in the original study [16]. Self-controlled clinical trials (the so-called quasi-experiments) were reported by four nonrandomized open label selected articles [25-28]. This type of study is not perfectly controlled and does not provide strong evidence and guidance for further clinical approaches; they are restricted to the analysis of timeseries and pre/postintervention analysis. However, they do contribute to extrapolate about the feasibility, safety, and tolerability of the clinical procedures. First of all, stroke patients who underwent mild therapeutic hypothermia (33$34^{\circ} \mathrm{C}$ ) reached the target temperature by either external or endovascular cooling methods. The range of latency to reach hypothermia threshold was in-between 3 and $9 \mathrm{~h}$, confirming the aforementioned feasibility studies. In terms of efficacy, it seems that hypothermia differentially affected stroke patients depending on the symptom severity. Acute ischemic stroke patients tolerated the procedure and presented clinical improvements in $24 \mathrm{~h}$ and at discharge $[25,26]$. Only one study reported postintervention mortality and it was relatively low for stroke standards $(\sim 10 \%)$. On the other hand, severe ischemic stroke patients had transient improvement in clinical symptoms (intracranial pressure, cerebral blood flow, and metabolism), but the effect tended to dissipate over a short follow-up period [27, 28]. Mortality rates were also higher ( $30 \%$ to $45 \%)$. Albeit the results are not statistically sound, at least in the investigational context, therapeutic hypothermia remains potentially promising, as a number of attempts using other neuroprotection strategies have previously failed $[10,42]$. It is necessary to evaluate the results of parallel-controlled clinical trials before drawing any conclusion, but hypothermia may contribute to positive outcomes, especially if not considered a stand-alone intervention, but rather an adjuvant procedure.

Eight studies designed as parallel-controlled clinical trials reached the criteria to be included in the present systematic review. Four of them were properly randomized and two blinded. None were a large multicenter clinical trial, but taking all studies together, we have a cohort of 288 individuals. Three nonrandomized studies used the conventional external cooling method. Two of them found out that the intracranial pressure did not change during hypothermia, and there were no difference in the stay duration in the hospital, no clinical improvement of stroke symptoms 3 months after discharge (mRS), and no difference in mortality ( $\sim 25 \%$ when reported) $[20,31]$. One study comparing mild therapeutic hypothermia versus hemicraniectomy surgery reached a different conclusion, finding clinical improvement in stroke symptoms (NIHSS) [29]. The same study found increased mortality in the hypothermic patients compared to hemicraniectomy (47\% versus $12 \%$ ) [35], which must be interpreted with caution. Historical control patients with malignant brain edema normally demonstrate a mortality rate around $70 \%$. This said, one should conclude that patients undergoing hemicraniectomy had better survival than those treated with hypothermia, but both are better than no treatment at all. It would not be right to consider that hypothermia increased mortality in this case, as there were no untreated controls [29]. One possible explanation for this difference in mortality is that treatment with hemicraniectomy is simply faster than cooling (particularly external cooling), so it could help patients within a narrow timeframe. One study employing endovascular cooling compared patients reaching hypothermic thresholds $\left(\sim 33^{\circ} \mathrm{C}\right)$ to those that could not bear such low temperatures (not below $35^{\circ} \mathrm{C}-36^{\circ} \mathrm{C}$ ) - only 7 out of 18 patients were effectively cooled [32]. This study found transient reduction in brain edema that vanished after 30 days and no significant difference in stroke symptoms at catheter removal or after 7to 30-day follow-up. Mortality was not reported [32].

Randomized controlled clinical trials studies report results of a relatively-large cohort of 127 patients (summedup), but unfortunately half of the studies associated hypothermia with surgical procedures affecting the interpretation 
of results. A single randomized study had clearer results comparing 18 hypothermic versus 22 control patients and did not find difference in stroke symptoms (per mRS and NIHSS), mean lesion growth, or mortality rate ( 25\%) [19]. The comparison of patients undergoing craniotomy with or without associated hypothermia induced by external cooling did not find difference in stroke symptoms (NIHSS) one to three days after surgery, and curiously, there were no deaths until discharge or at follow-up [30]. The interpretation of these results is difficult as the effect of the craniotomy surgery itself is a strong confounding factor. For example, this was the only study where the mortality rate was absent, which may speak for a ceiling effect of the surgical procedure that may not have been potentiated by hypothermia. Controversially, another randomized study comparing craniectomy patients with and without hypothermia found a trend towards statistical significance in stroke symptoms (NIHSS and BI) in the 6-month follow-up phase [34]. A relatively low mortality rate was again reported $(\sim 10 \%)$, although with no statistical difference between groups, which emphasize that this must have been due to the surgical effect rather than due to hypothermia [34].

The more recent and largest study of in awake stroke patients employing endovascular cooling and antishivering and thrombolytic adjuvant therapies shall be used as a reference in the field [33]. It performed clear comparisons between well-selected groups of patients against a clear control group and confirmed the earlier observations with stronger evidence. No difference was observed at day 30 or 90 after intervention using NIHSS or mRS. Moreover, there was no apparent difference on clinical outcome or occurrence of adverse events regarding time of intervention and tPA $(<3 \mathrm{~h}$ versus 3-6 h of symptoms onset). Mortality was comparable between groups and pneumonia occurred more frequently in hypothermic patients than in controls.

The effects of hypothermia on stroke aftermath are still a matter of interest as illustrated by the fact that a large randomized clinical trial is currently ongoing. In this study, the "Intravascular Cooling in the Treatment of Stroke 2/3 (ICTus 2/3) Trial" plans to enroll 400 patients and investigate the effects of combining cooling with thrombolytic therapy (tPA) in a randomized clinical trial design. Control groups will receive tPA and kept with normal body temperature.

The putative neuroprotective effects of profound hypothermia have been reported in the literature, but they largely rely on case reports, truncated study designs, or animal models. There were previous reports of strong beneficial effects of hypothermia-induced neuroprotection in cerebral ischemia, reducing the infarct volumes up to $90 \%$ in rodents [13-15], which some expected to extrapolate to human patients. However, this assumption is not confirmed by the current systematic review decrypting details of the study designs and outcomes. Earlier animal model research has consistently shown that hypothermia reduces cerebral metabolism, decreases levels of excitatory aminoacids, stabilizes the blood-brain barrier, and decreases inflammatory markers after brain injury $[12,43,44]$. Therefore, this review article should not be used as an argument to question the results in animal models of stroke, but rather to emphasize that the results of translational research are not always obvious and predictable. On the other hand, we confirm the view that high temperatures on admission may worsen stroke symptoms and long-term outcomes. Our suggestion is that bodily temperature control might (and should) be used to prevent the effects of hyperthermia, rather than to induce hypothermia. Apart from its putative application on stroke, the use of hypothermia for the therapy of neuronal injuries in general was already kept relatively aside due to patient discomfort and side effects such as shivering, infections, and coagulations disturbances [45].

The "classic" contention that low admission body temperature seems to improve short-term survival and neurological recovery is still valid [7], but it seems that the therapeutic application of hypothermia on stroke patients is still unrealistically overemphasized. One possibility is that body temperature plays a strong influence on the very early steps of stroke physiopathology (i.e., within the very first hours of symptoms onset) but acts in a restricted time window that precludes taking advantage of these effects in a regular clinical setting. The relatively recent development of a cooling helmet may foster the development of a faster brain cooling method to catch up with this therapeutic window [36], but to the best of our knowledge, no successful clinical application was yet reported in the scientific literature.

In summary, we did not find conclusive evidence supporting the use of mild therapeutic hypothermia on stroke patients in hospital settings, although strong evidence suggests that body temperature influences stroke symptomatology. At least one large randomized controlled clinical trial is still necessary to conclude on this subject and finally guide patient management. The use of rapid cooling techniques may result in more promising results due to an apparent relatively narrow time-window of therapeutic opportunity for hypothermia-induced neuroprotection on stroke.
Abbreviations
BI: $\quad$ Barthel index
CENTRAL: Cochrane Central Register of Controlled Trials
mRS: $\quad$ Modified Rankin scale
NIHSS: $\quad$ NIH Stroke Scale
QUOROM: Quality of reporting of meta-analyses.

\section{Conflict of Interests}

The authors declare that they have no Conflict of interests.

\section{Authors' Contributions}

All authors participated in the preparation of the manuscript and read and approved the final manuscript.

\section{References}

[1] V. L. Feigin, "Stroke epidemiology in the developing world," Lancet, vol. 365, no. 9478, pp. 2160-2161, 2005. 
[2] G. A. Donnan, M. Fisher, M. Macleod, and S. M. Davis, "Stroke," The Lancet, vol. 371, no. 9624, pp. 1612-1623, 2008.

[3] G. Azzimondi, L. Bassein, F. Nonino et al., "Fever in acute stroke worsens prognosis: a prospective study," Stroke, vol. 26, no. 11, pp. 2040-2043, 1995.

[4] D. Corbett and J. Thornhill, "Temperature modulation (hypothermic and hyperthermic conditions) and its influence on histological and behavioral outcomes following cerebral ischemia," Brain Pathology, vol. 10, no. 1, pp. 145-152, 2000.

[5] L. P. Kammersgaard, H. S. Jørgensen, J. A. Rungby et al., "Admission body temperature predicts long-term mortality after acute stroke: the Copenhagen Stroke Study," Stroke, vol. 33, no. 7, pp. 1759-1762, 2002.

[6] P. Meden, K. Overgaard, H. Pedersen, and G. Boysen, "The influence of body temperature on infarct volume and thrombolytic therapy in a rat embolic stroke model," Brain Research, vol. 647, no. 1, pp. 131-138, 1994.

[7] J. Reith, H. S. Jørgensen, P. M. Pedersen et al., "Body temperature in acute stroke: relation to stroke severity, infarct size, mortality, and outcome," Lancet, vol. 347, no. 8999, pp. 422425, 1996.

[8] M. K. Roy and A. Ray, "Effect of body temperature on mortality of acute stroke," Journal of Association of Physicians of India, vol. 52, pp. 959-961, 2004.

[9] Y. Wang, L. L. Y. Lim, C. Levi, R. F. Heller, and J. Fisher, "Influence of admission body temperature on stroke mortality," Stroke, vol. 31, no. 2, pp. 404-409, 2000.

[10] V. E. O’Collins, M. R. Macleod, G. A. Donnan, L. L. Horky, B. H. Van Der Worp, and D. W. Howells, "1,026 Experimental treatments in acute stroke," Annals of Neurology, vol. 59, no. 3, pp. 467-477, 2006.

[11] Y. Kim, R. Busto, W. D. Dietrich, S. Kraydieh, and M. D. Ginsberg, "Delayed postischemic hyperthermia in awake rats worsens the histopathological outcome of transient focal cerebral ischemia," Stroke, vol. 27, no. 12, pp. 2274-2281, 1996.

[12] M. D. Ginsberg, L. L. Sternau, M. Y. Globus, W. D. Dietrich, and R. Busto, "Therapeutic modulation of brain temperature: relevance to ischemic brain injury," Cerebrovascular and Brain Metabolism Reviews, vol. 4, no. 3, pp. 189-225, 1992.

[13] H. Karibe, J. Chen, G. J. Zarow, S. H. Graham, and P. R. Weinstein, "Delayed induction of mild hypothermia to reduce infarct volume after temporary middle cerebral artery occlusion in rats," Journal of Neurosurgery, vol. 80, no. 1, pp. 112119,1994

[14] E. Morikawa, M. D. Ginsberg, W. D. Dietrich et al., "The significance of brain temperature in focal cerebral ischemia: histopathological consequences of middle cerebral artery occlusion in the rat," Journal of Cerebral Blood Flow and Metabolism, vol. 12, no. 3, pp. 380-389, 1992.

[15] D. Xue, Z. G. Huang, K. E. Smith, and A. M. Buchan, "Immediate or delayed mild hypothermia prevents focal cerebral infection," Brain Research, vol. 587, no. 1, pp. 66-72, 1992.

[16] C. Ikonomidou, J. L. Mosinger, and J. W. Olney, "Нypothermia enhances protective effect of MK-801 against hypoxic/ ischemic brain damage in infants rats," Brain Research, vol. 487, no. 1, pp. 184-187, 1989.

[17] T. M. Hemmen and P. D. Lyden, "Induced hypothermia for acute stroke," Stroke, vol. 38, no. 2, pp. 794-799, 2007.

[18] A. G. Doufas, C. M. Lin, M. I. Suleman et al., "Dexmedetomidine and meperidine additively reduce the shivering threshold in humans," Stroke, vol. 34, no. 5, pp. 1218-1223, 2003.

[19] M. A. De Georgia, D. W. Krieger, A. Abou-Chebl et al., "Cooling for acute ischemic brain damage (COOL AID): a feasibility trial of endovascular cooling," Neurology, vol. 63, no. 2, pp. 312-317, 2004.

[20] D. W. Krieger, M. A. De Georgia, A. Abou-Chebl et al., "Cooling for acute ischemic brain damage (COOL AID): an open pilot study of induced hypothermia in acute ischemic stroke," Stroke, vol. 32, no. 8, pp. 1847-1854, 2001.

[21] S. A. Mayer, R. G. Kowalski, M. Presciutti et al., "Clinical trial of a novel surface cooling system for fever control in neurocritical care patients," Critical Care Medicine, vol. 32, no. 12, pp. 2508-2515, 2004.

[22] R. M. Zweifler, M. E. Voorhees, M. A. Mahmood, and D. D. Alday, "Induction and maintenance of mild hypothermia by surface cooling in non-intubated subjects," Journal of Stroke and Cerebrovascular Diseases, vol. 12, no. 5, pp. 237-243, 2003.

[23] S. E. Kasner, T. Wein, P. Piriyawat et al., "Acetaminophen for altering body temperature in acute stroke: a randomized clinical trial," Stroke, vol. 33, no. 1, pp. 130-134, 2002.

[24] H. M. den Hertog, H. B. van der Worp, H. M. A. van Gemert et al., "The Paracetamol (Acetaminophen) In Stroke (PAIS) trial: a multicentre, randomised, placebo-controlled, phase III trial," The Lancet Neurology, vol. 8, no. 5, pp. 434-440, 2009.

[25] R. Kollmar, P. D. Schellinger, T. Steigleder, M. Köhrmann, and S. Schwab, "Ice-cold saline for the induction of mild hypothermia in patients with acute ischemic stroke: a pilot study," Stroke, vol. 40, no. 5, pp. 1907-1909, 2009.

[26] S. Martin-Schild, H. Hallevi, H. Shaltoni et al., "Combined neuroprotective modalities coupled with thrombolysis in acute ischemic stroke: a pilot study of caffeinol and mild hypothermia," Journal of Stroke and Cerebrovascular Diseases, vol. 18, no. 2, pp. 86-96, 2009.

[27] E. Keller, T. Steiner, J. Fandino, S. Schwab, and W. Hacke, "Changes in cerebral blood flow and oxygen metabolism during moderate hypothermia in patients with severe middle cerebral artery infarction," Neurosurgical Focus, vol. 8, no. 5, p. e4, 2000 .

[28] S. Schwab, S. Schwarz, A. Aschoff, E. Keller, and W. Hacke, "Moderate hypothermia and brain temperature in patients with severe middle cerebral artery infarction," Acta Neurochirurgica, Supplement, vol. 1998, no. 71, pp. 131-134, 1998.

[29] S. Schwab, S. Schwarz, M. Spranger, E. Keller, M. Bertram, and W. Hacke, "Moderate hypothermia in the treatment of patients with severe middle cerebral artery infarction," Stroke, vol. 29, no. 12, pp. 2461-2466, 1998.

[30] B. J. Hindman, M. M. Todd, A. W. Gelb et al., "Mild hypothermia as a protective therapy during intracranial aneurysm surgery: a randomized prospective pilot trial," Neurosurgery, vol. 44, no. 1, pp. 23-33, 1999.

[31] S. Horstmann, J. A. Koziol, F. Martinez-Torres, S. Nagel, H. Gardner, and S. Wagner, "Sonographic monitoring of mass effect in stroke patients treated with hypothermia. Correlation with intracranial pressure and matrix metalloproteinase 2 and 9 expression," Journal of the Neurological Sciences, vol. 276, no. 1-2, pp. 75-78, 2009.

[32] K. Z. Guluma, H. Oh, S. W. Yu, B. C. Meyer, K. Rapp, and P. D. Lyden, "Effect of endovascular hypothermia on acute ischemic edema: morphometric analysis of the ICTuS trial," Neurocritical Care, vol. 8, no. 1, pp. 42-47, 2008.

[33] T. M. Hemmen, R. Raman, K. Z. Guluma et al., "Intravenous thrombolysis plus hypothermia for acute treatment of ischemic stroke (ICTuS-L): final results," Stroke, vol. 41, no. 10, pp. 2265-2270, 2010.

[34] T. Els, E. Oehm, S. Voigt, J. Klisch, A. Hetzel, and J. Kassubek, "Safety and therapeutical benefit of hemicraniectomy combined with mild hypothermia in comparison with 
hemicraniectomy alone in patients with malignant ischemic stroke," Cerebrovascular Diseases, vol. 21, no. 1-2, pp. 79-85, 2006.

[35] D. Georgiadis, S. Schwarz, A. Aschoff, and S. Schwab, "Hemicraniectomy and moderate hypothermia in patients with severe ischemic stroke," Stroke, vol. 33, no. 6, pp. 1584-1588, 2002.

[36] H. Wang, W. Olivero, G. Lanzino et al., "Rapid and selective cerebral hypothermia achieved using a cooling helmet," Journal of Neurosurgery, vol. 100, no. 2, pp. 272-277, 2004.

[37] D. Georgiadis, S. Schwarz, R. Kollmar, and S. Schwab, "Endovascular cooling for moderate hypothermia in patients with acute stroke: first results of a novel approach," Stroke, vol. 32, no. 11, pp. 2550-2553, 2001.

[38] P. D. Lyden, R. L. Allgren, K. Ng et al., "Intravascular cooling in the treatment of stroke (ICTuS): early clinical experience," Journal of Stroke and Cerebrovascular Diseases, vol. 14, no. 3, pp. 107-114, 2005.

[39] A. Abou-Chebl, M. A. DeGeorgia, J. C. Andrefsky, and D. W. Krieger, "Technical refinements and drawbacks of a surface cooling technique for the treatment of severe acute ischemic stroke," Neurocritical Care, vol. 1, no. 2, pp. 131-143, 2004.

[40] T. Steiner, T. Friede, A. Aschoff, P. D. Schellinger, S. Schwab, and W. Hacke, "Effect and feasibility of controlled rewarming after moderate hypothermia in stroke patients with malignant infarction of the middle cerebral artery," Stroke, vol. 32, no. 12, pp. 2833-2835, 2001.

[41] K. Z. Guluma, T. M. Hemmen, S. E. Olsen, K. S. Rapp, and P. D. Lyden, "A trial of therapeutic hypothermia via endovascular approach in awake patients with acute ischemic stroke: methodology," Academic Emergency Medicine, vol. 13, no. 8, pp. 820-827, 2006.

[42] S. E. Lakhan, A. Kirchgessner, and M. Hofer, "Inflammatory mechanisms in ischemic stroke: therapeutic approaches," Journal of Translational Medicine, vol. 7, article 97, 2009.

[43] J. Maher and V. Hachinski, "Hypothermia as a potential treatment for cerebral ischemia," Cerebrovascular and Brain Metabolism Reviews, vol. 5, no. 4, pp. 277-300, 1993.

[44] K. Nakashima and M. M. Todd, "Effects of hypothermia on the rate of excitatory amino acid release after ischemic depolarization," Stroke, vol. 27, no. 5, pp. 913-918, 1996.

[45] P. Safar, M. Klain, and S. Tisherman, "Selective brain cooling after cardiac arrest," Critical Care Medicine, vol. 24, no. 6, pp. 911-914, 1996. 


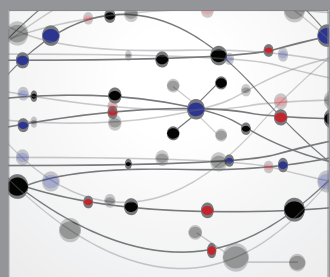

The Scientific World Journal
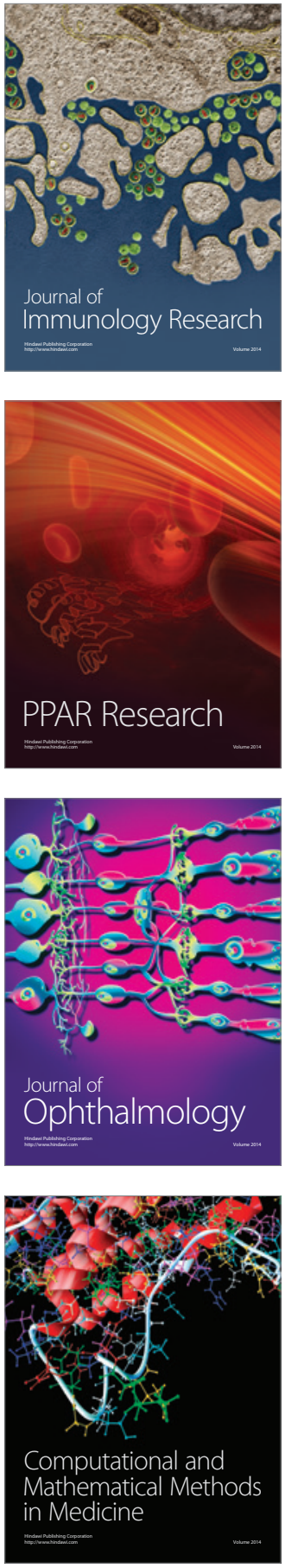

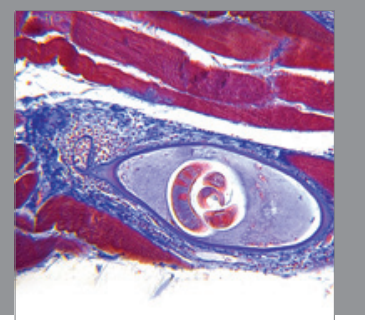

Gastroenterology

Research and Practice
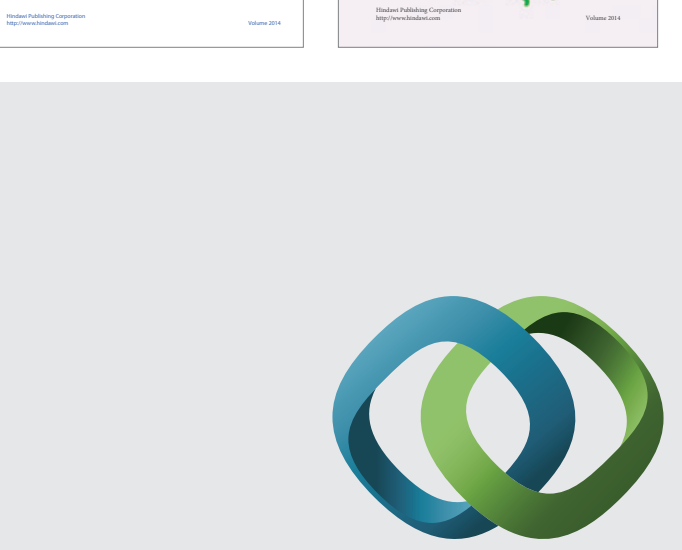

\section{Hindawi}

Submit your manuscripts at

http://www.hindawi.com
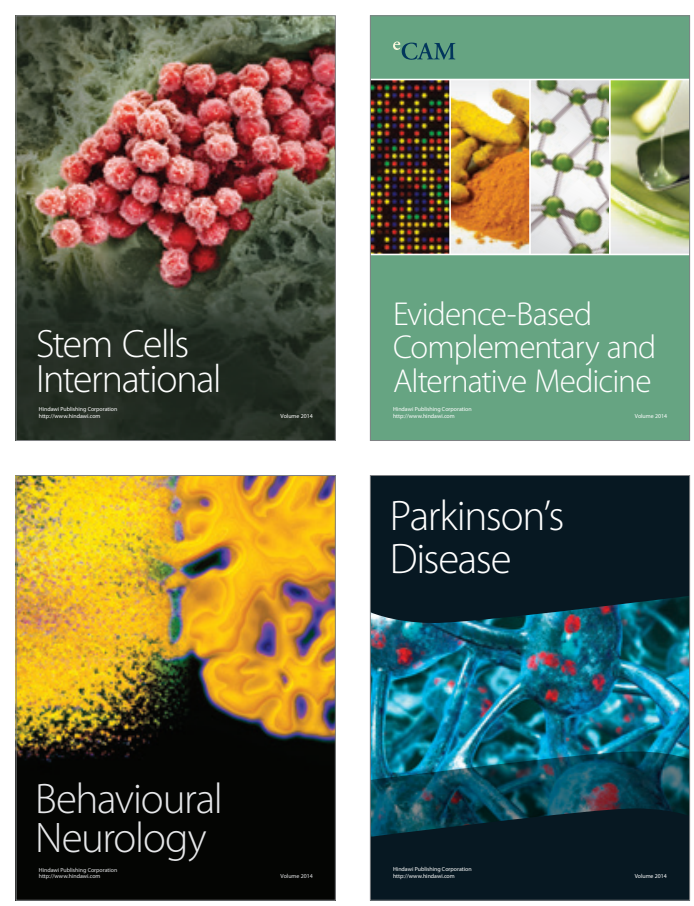

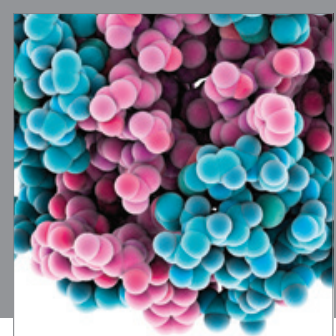

Journal of
Diabetes Research

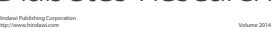

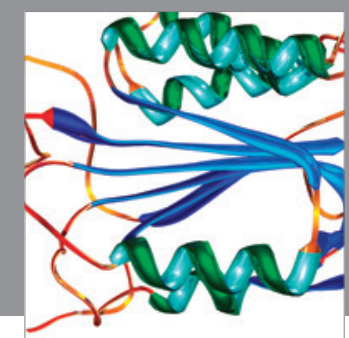

Disease Markers
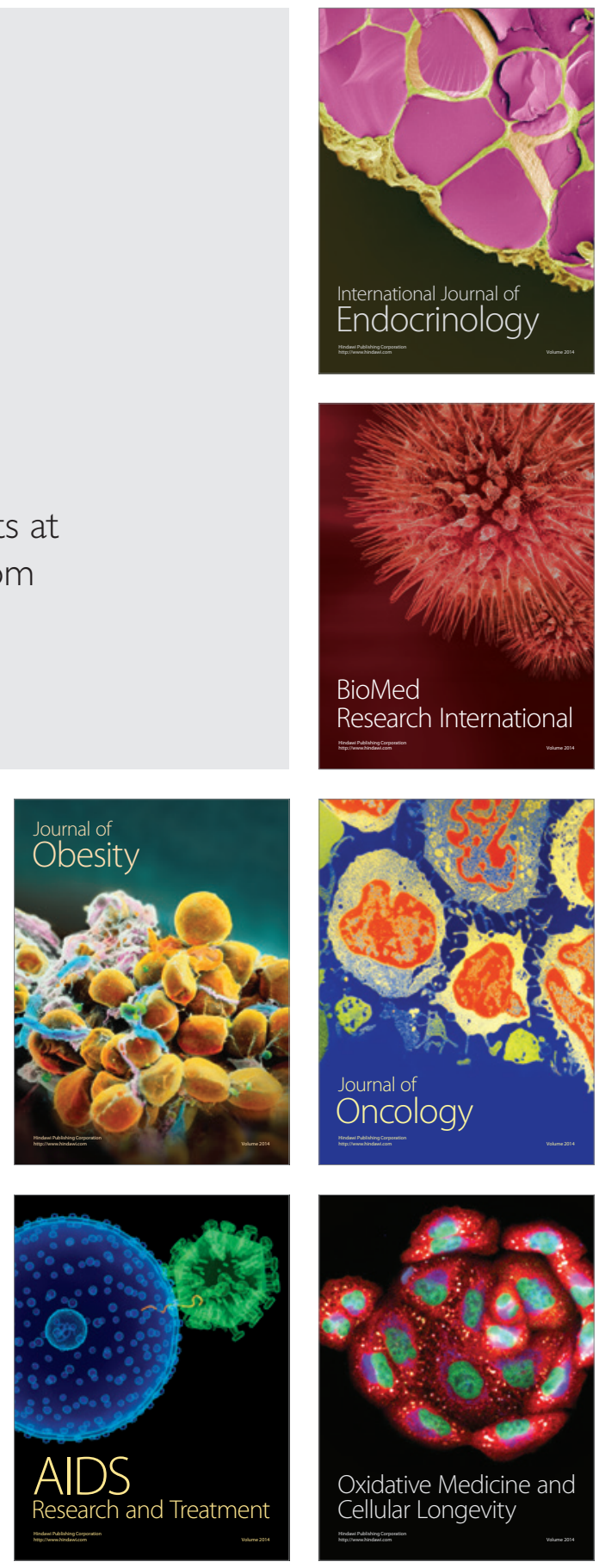